\title{
Methods for Determining Aircraft Surface State at Lesser-Equipped Airports
}

\author{
Keenan Roach ${ }^{1}$ and Jody Null ${ }^{2}$ \\ UARC/NASA Ames Research Center, Ft. Worth, TX, 76155
}

\begin{abstract}
Tactical departure scheduling within a terminal airspace must accommodate a wide spectrum of surveillance and communication capabilities at multiple airports. The success of such a scheduler is highly dependent upon the knowledge of a departure's state while it is still on the surface. Airports within a common Terminal RAdar CONtrol (TRACON) airspace possess varying levels of surface surveillance infrastructure which directly impacts uncertainties in wheels-off times. Large airports have access to surface surveillance data, which is shared with the TRACON, while lesser-equipped airports still rely solely on controllers in Air Traffic Control Towers (Towers). Coordination between TRACON and Towers can be greatly enhanced when the TRACON controller has access to the surface surveillance and the associated decision-support tools at well-equipped airports. Similar coordination at lesser-equipped airports is still based on verbal communications. This paper investigates possible methods to reduce the uncertainty in wheels-off time predictions at the lesser-equipped airports through the novel use of Over-the-Air (OTA) data transmissions. We also discuss the methods and equipment used to collect sample data at lesser-equipped airports within a large US TRACON, as well as the data evaluation to determine if meaningful information can be extracted from it.
\end{abstract}

\section{Introduction}

$\mathrm{N}$ ASA's Airspace Technology Demonstration sub-project 2 (ATD-2) will develop and evaluate tools for Integrated Arrival/Departure/Surface (IADS) operations to meet the goals of the Next Generation air transportation system (NextGen). ${ }^{1}$ One part of this integration involves the coordination between airport Air Traffic Control Towers (Towers) and Air Route Traffic Control Center (Center) Traffic Management Units (TMUs) regarding tactical departure release times. Departures wishing to join a constrained overhead stream of traffic are routinely held on the surface until an available slot is found or made available by controllers. This type of Traffic Management Initiative (TMI) is known as a Call For Release (CFR) procedure. Recent research and development, namely the Precision Departure Release Capability (PDRC), has demonstrated the value in using surface trajectorybased takeoff time predictions for constrained departure scheduling. ${ }^{2,3,4}$

Besides validating the concept of a Tactical Departure Scheduler (TDS), the success of PDRC also revealed the need to apply TDS technology to other areas of departure operations. While PDRC focused on Tower to Center communications for departures from well-equipped airports bound for a constrained en route stream, CFRs between the Tower and Terminal RAdar CONtrol (TRACON) were left unchanged. These departure boundary CFRs affect multiple airports within a common terminal area, with varying levels of surface surveillance, and impart substantial delay and uncertainty to departure operations. ${ }^{5}$ Departures within a TRACON typically use a common set of departure fixes regardless of the originating airport, and during times when a fix is constrained, aircraft from different airports must be scheduled over the fix by using coordinated surface release times. Rectifying the imbalance in technology between well and lesser-equipped airports has been the focus of recent work ${ }^{6}$; however, a suitable substitute, mainly because of the prohibitive cost, for the high fidelity surface surveillance used at the wellequipped airports has not yet been found for use at the lesser-equipped airports.

Surface surveillance is the key to the success of scheduling departures while they are still on the ground because it reduces uncertainty in predicted departure times ${ }^{5}$. Well-equipped airports have Airport Surface Detection Equipment, Mode X (ASDE-X) from which surface trajectories are generated. Future technologies plan on using

\footnotetext{
${ }^{1}$ Research Engineer, NASA/FAA North Texas Research Station, AIAA Member.

${ }^{2}$ Software/Systems Engineer, NASA/FAA North Texas Research Station, AIAA Member.
}

1

American Institute of Aeronautics and Astronautics 
self-reporting Automatic Dependent Surveillance - Broadcast (ADS-B) messages for surface data or the Aeronautical Mobile Airport Communications System (AeroMACS) ${ }^{7}$ for aggregating and disseminating multilateration data from non-ASDE-X sources. However, only a minority of aircraft at lesser-equipped airports use ADS-B today and AeroMACS requires communication infrastructure. Use of higher fidelity systems is more a matter of equipage rather than choice. This paper presents a novel approach to surface surveillance at lesserequipped airports, which uses Mode $\mathrm{S}$ transponder messages as a means to determine the state of departures while they are still on the ground. Knowing the surface state of departures at lesser-equipped airports will allow them to be integrated into a tactical departure scheduler.

\section{Current TRACON Departure Operations}

Many TRACONs in the National Airspace System (NAS) serve multiple airports of varied sizes and equipage levels. TRACON controllers must balance the demand of larger airports with the smaller lesser-equipped airports and maintain equitable use of the airspace at the same time. This is true for the Dallas/Fort Worth (DFW) D10 TRACON; and as shown in Figure 1, many airports lie within the boundary of D10, but two are the focus of this paper. Dallas Love Field (DAL) is designated because it was the subject of this study, while DFW is denoted because it is the largest airport within D10. The remaining airport locations are shown in order to give additional context to the terminal area. During nominal conditions the sixteen departure fixes provide adequate bandwidth to handle all the airports without introducing departure delay. However, during off-nominal times, such as due to severe weather, certain departure fixes are shut down causing the demand to exceed throughput for the remaining open fixes. When this occurs the TRACON will initiate a Call for Release (CFR) restriction on departures headed toward the affected fixes. The CFR initiative, also known as an Approval Request (APREQ), allows the departure controller to meter the departures over a specific fix by regulating the release times for affected departures from all

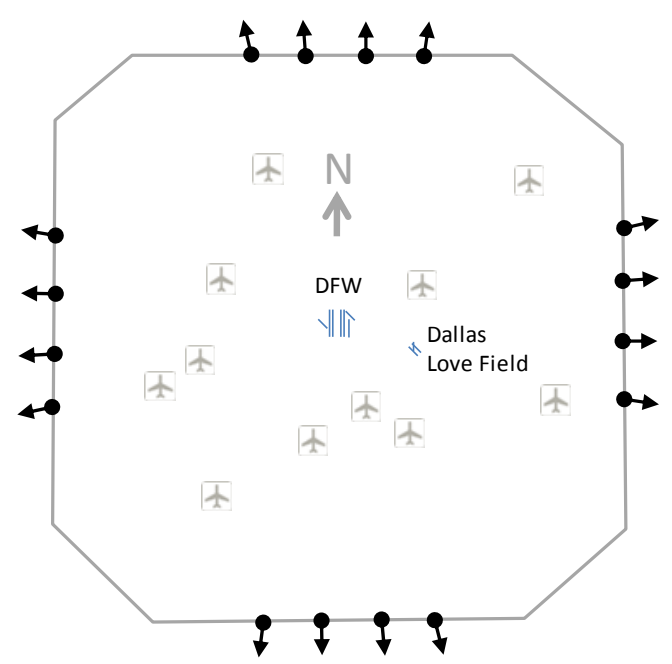

Figure 1. The DFW D10 TRACON. Airports are shown within the boundary line. The 16 dots are the departure fixes with arrows showing the exit direction from the TRACON. airports.

CFRs are usually a result of a Miles-In-Trail (MIT) restriction on a downstream fix. For the PDRC evaluation, this fix was in the Center's airspace, and the release time had to be coordinated between Center and tower controllers. In the terminal area, the departure fix is on the TRACON boundary and the coordination is handled between the TRACON and tower controllers. In both cases, the controller responsible for the fix has to regulate the flow of aircraft by adding delay to departures on the ground.

The largest cause of delay, that has the potential to be minimized, during a CFR restriction is a result of departure uncertainty in terms of predicted off times and fix crossing times ${ }^{6,8}$. Controllers must merge multiple restricted departures from different airports over available fixes. Most D10 departures originate from DFW, as it is the largest airport in the TRACON, so it is the easiest to categorize the departures as DFW and non-DFW. Ideally, restricted DFW and non-DFW flights would depart according to a common tactical scheduler. The release time (the time a departure begins its takeoff roll) would be synchronized so that the constraint at the TRACON boundary fix would be satisfied while minimizing the on-ground delay. For example, two airports may be able to release departures at the same time if their geographic locations would naturally separate the aircraft when they reach the fix.

Unfortunately, in current operations, the wheels-off time uncertainty is too large at the lesser-equipped airports to schedule departures seamlessly. This forces controllers to operate in an airport-by-airport manner instead of a coordinated TRACON level. Restricted flights from one airport are released while all others are held on the ground waiting their turn. Compartmentalizing departures by airport maintains safety and ensures separation at the fix, but it is also the main source of delay that a scheduler seeks to avoid. Holding an aircraft on the ground at a lesserequipped airport while allowing several flights from DFW depart will add delay to only one aircraft, but conversely, several aircraft at DFW will accrue delay waiting for one departure at a lesser-equipped airport. 
The technological imbalance between DFW and the other lesser-equipped airports in the TRACON is what makes integrating all departures into a common scheduler so challenging. The tactical departure scheduler at DFW, the only D10 airport with surface surveillance, uses ASDE-X to generate surface trajectories which reduce the predicted off time uncertainty. The lesser-equipped airports still verbally communicate the need for a departure clearance to the TRACON controller once a CFR flight has requested it. At the TRACON or system level very little is known about the readiness level or earliest possible takeoff time of restricted departures from lesser-equipped airports. The data flow in chronological order is as follows: the filed flight plan information is received at the Tower, the Tower requests clearance for the departure, the TRACON grants clearance once a slot has been made, and finally the first in-air track hit is sent to a TRACON controller's radar screen. The verbal communications do not contain any precise information that could be used to accurately schedule the departure. Only the in-air track hit provides accurate location, time, and identification, but by that point departure slot scheduling becomes irrelevant as it must be done on the surface.

\section{Departure Constraints in the Terminal Airspace}

When the throughput capacity at the boundary of the Terminal area is diminished, departures are restricted in order to maintain adequate separation. The causes of these constraints can originate at the boundary itself or further downstream. Figure 2 shows the four most common scenarios that lead to departure restrictions. In subfigure A, three of the east side departure fixes are closed due to a convective weather cell. Combining routes from one or more fixes to another fix on the same gate (each side of the TRACON is called a gate and contains four departure fixes) is known as a departure fix compression. While convective weather is often the trigger for this event, other environmental and traffic situations can lead to a fix compression. It is common for a 20 MIT restriction to be placed on a fix under these conditions due to uncertainty in fix crossing times and weather movement.

Subfigure B depicts a departure fix swap, in which a flight's departure fix is changed from one TRACON gate to another. A swap may occur when a gate is only partially closed, or as the figure illustrates, with the entire closure of a gate. Unlike fix compressions, which keep flights on the same gate, a swap requires a flight plan amendment to account for differences in fuel consumption. Again, 20 MIT is common in this situation, but the severity of the restriction is dependent upon the particular circumstances of each event. A TRACON departure fix swap should not be confused with a Severe Weather Avoidance Program (SWAP), which is an en route traffic management initiative.

There are often traffic management initiatives that originate outside of the terminal area. Flow restrictions propagate back and impact local departure fixes. Depending on their type, these downstream constraints (subfigure C) can restrict one or more fixes on a gate. Unlike the fix compressions and swaps, which impact all departures using the fix, these constraints affect only the departures bound for the specified downstream restriction. This requires the controller to merge restricted and nonrestricted departures over the same fix.

The final case (subfigure D) is one in which there is high demand from multiple airports over one fix. This scenario usually occurs during a heavy, or banked, departure push out of DFW. These times of high departure demand lead to
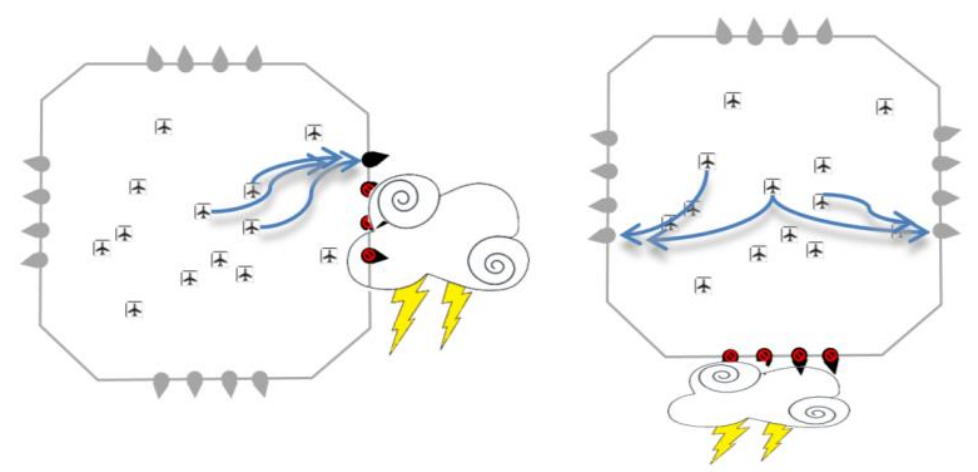

A. Departure Fix Compression

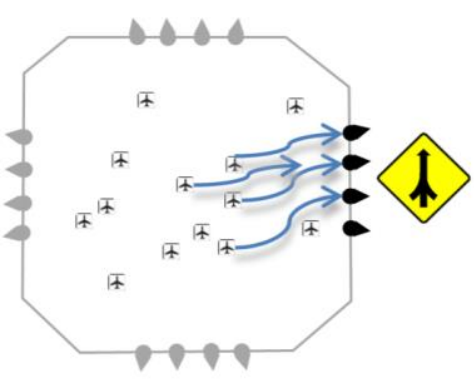

C. Downstream Constraint
B. Departure Fix Swap

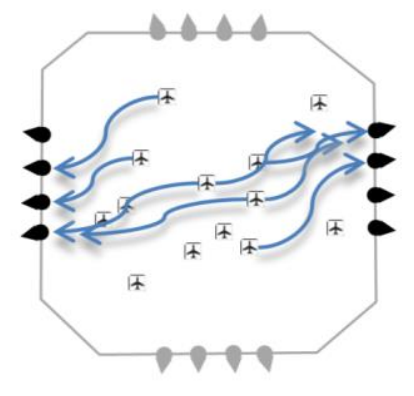

D. High Demand

Figure 2. Types of departure constraints in the D10 TRACON. Each subplot of D10 shows a scenario in which affected departures would be put under a Call for Release restriction. 
restricted use of departure fixes to ensure safe separation. Accurate predictions and automation can be helpful in calculating future demand over a fix, but only DFW and DAL have Area Navigation (RNAV) departure routes within the TRACON. RNAV routes reduce the uncertainty in trajectory predictions because of their rigid structure specifying equipage for a $1 \mathrm{~nm}$ navigation precision'. The lesser-equipped airports, as well as all aircraft without the required equipment to fly RNAV routes, still rely on vectored control. Vectored routes contain more uncertainty in terms of trajectory prediction and therefore require greater separation when being merged over a fix with other vectored and RNAV departures.

\section{Currently Available Surveillance Data}

The main objective of this research is to reduce the imbalance in technology between well and lesser-equipped airports with regard to surface surveillance data and departure scheduling. In this context, this specific work aims to increase the early awareness of departures on the surface at lesser-equipped airports in order to improve the efficiency of tactical departure scheduling within a metroplex environment. Knowing when an aircraft pushes back from the gate or becomes active is a key piece of information needed for an integrated terminal area departure scheduler. Without the surface surveillance data, the lesser-equipped airports need an alternate method for determining a departure's state with regard to its surface activity. The novel use of Over-the-Air (OTA) aircraft transmissions and pilot/controller radio communications are two examples of existing data feeds that are currently available, but not leveraged to their fullest potential. Using existing data is the logical first step toward a TRACONwide surrogate surveillance solution.

PASSUR Aerospace recently installed infrastructure at DAL allowing them to use multilateration algorithms to determine an aircraft's surface position based on its Mode S transmissions. The aircraft positions are fused together with various other feeds including air carrier and flight plan data. The integration of these data sources makes the data easy to use from a researcher's perspective. Because the PASSUR data contains the most complete surface picture at DAL currently available, four months of data were purchased and considered "baseline data" for evaluation and validation of other lower level forms of data like the Mode $S$ messages. The PASSUR data contained the fields shown in Table 1.

Table 1 - PASSUR data fields.

\begin{tabular}{|c|c|}
\hline Flight ID & the ID assigned by the air carriers to its planes or tail number of general aviation planes \\
\hline Beacon Code & the decimal version of the 4 digit octal code transmitted by the Mode $\mathrm{A} / \mathrm{C}$ transponder \\
\hline Tail Number & the tail number of the aircraft, also known as the registration number \\
\hline \multicolumn{2}{|l|}{ Aircraft Type } \\
\hline \multicolumn{2}{|l|}{ Destination Airport } \\
\hline \multicolumn{2}{|l|}{ Departure Gate Number } \\
\hline Scheduled Departure Time & the scheduled gate push back time \\
\hline Actual Gate Out Time & the actual gate push back time \\
\hline Actual Departure Time & wheels-off time \\
\hline 1st Mode S Time & the time the first Mode $\mathrm{S}$ message was received \\
\hline Departure Runway & runway used by the departing aircraft \\
\hline Runway Queue Time & the time the aircraft enters the departure queue area \\
\hline Runway Time & the time the aircraft crosses the departure runway threshold \\
\hline Aircraft Position & latitude, longitude, and time \\
\hline
\end{tabular}

Of all the data transmissions originating from an aircraft, transponder data provide the greatest coverage in terms of data availability and they are the most continuous once the aircraft becomes active. The data is broadcast overthe-air and is freely available to any antenna within line-of-sight of the aircraft. For these reasons, and the fact that aircraft begin sending transponder data while on the surface, leveraging the transponder data feed for determination of an 'active on surface' state for departures at lesser-equipped airports is a sensible approach.

Transponders can be broken into two groups; Mode A/C and Mode S. Mode A/C is part of the Secondary Surveillance Radar (SSR) system developed in World War II to determine friend or foe aircraft. In today's civil 
airspace, aircraft broadcast (or squawk) a particular beacon code which is received by a ground station. This effectively links the position of the aircraft with an identification beacon. The ' $\mathrm{C}$ ' component of Mode $\mathrm{A} / \mathrm{C}$ provides the aircraft's altitude to the ground station. The beacon codes and altitudes are both sent in the same format, a four digit octal number.

Mode $\mathrm{A} / \mathrm{C}$ messages pose some difficulties in deciphering what type of message is being broadcast. The ground station interrogator requests either beacon codes or altitudes, so it will know what message type to expect as a response. Those ground stations only capable of receiving these messages must further filter them using other means. The method by which the Mode A/C messages were filtered in this research is described in a later section.

Mode S messages are an enhanced version of the Mode $\mathrm{A} / \mathrm{C}$ system that allows selective call and respond messages between ground-based interrogation stations and aircraft. Mode $\mathrm{S}$ was developed because of several limitations with Mode A/C, including the limited number of beacon codes (4096 total with many reserved for special uses) and the possibility of unwanted replies originating from aircraft in another ground station's airspace overlapping the desired messages. Every aircraft with a Mode S system has its own unique Mode S address, a hexadecimal code that is algorithmically generated based on the registration number, in contrast to the sharing of codes in the Mode A/C system.

Automatic Dependence Surveillance Data (ADS-B) is a sub message that is embedded in an extended version of the Mode S message. ADS-B data contains the real-time latitude and longitude information for a flight, in addition to other automatically broadcast information dependent on the aircraft's navigation system. ADS-B data is a cornerstone of NextGen air traffic modernization, but it is not mandated for use in airspace currently requiring a Mode C transponder until the year $2020^{10}$. Current ADS-B participation greatly varies between commercial carriers as well as within the general aviation community. In the future, ADS-B will be a more comprehensive solution to surface surveillance at lesser-equipped airports, but today the gaps in coverage are too great for it to be a viable option.

Other data sources not used in this study were the Aircraft Communications Addressing and Reporting System (ACARS) and audio communications between pilot and controller. ACARS was originally developed as a semiautomated communication system between airline dispatchers and aircraft. The system allows pilots and dispatchers to send text messages back and forth at their discretion. The system also sends automated status updates that are triggered by events, such as 'doors closed,' 'gear up,' 'pushback,' etc. While this data (specifically the 'pushback' message) would be perfect for use as a surrogate surface surveillance data source, the availability is extremely lacking. At DAL, using ACARS is not a viable option because only airlines use the service and general aviation aircraft make up a significant mix of aircraft there and at other lesser-equipped airports. Unlike ADS-B, which has a compliance mandate, there is no indication that ACARS will be more widely used in the future.

The verbal communications between pilots and controllers reveal precisely when a plane requests clearance to enter a taxiway. Industry has worked on speech recognition in the past ${ }^{11}$; however, the success of using speech recognition software to accurately transcribe verbal communications into usable data is not high enough to be a standalone solution. Due to variability in dialect and pronunciation, the current system can be trained to identify only a small set of key words with a high degree of confidence. Even with this limited vocabulary there are challenges. At the smaller airports it is common practice for the ground controller to refer to an aircraft by the last two alphanumeric characters of its tail number. There are cases when more than one aircraft have the same two ending characters on their tail numbers, so even with a perfectly working speech decoder, additional data sources would still be needed to positively identify aircraft.

\section{Collecting Dallas Love Field Data}

While transponder data is broadcast for all to 'hear', only those able to decode the messages can use it. In addition to its intended use, many plane spotters and aviation enthusiasts use hobbyist grade equipment to decipher transponder messages. Most hobbyists use the ADS-B data to plot airborne aircraft tracks. This works out quite well as the field of view from a roof-mounted antenna to aircraft in the sky is excellent. Collecting surface-tosurface messages is more challenging and requires the antenna to be in close proximity to the intended target to avoid any obstructions. As DAL was the main focus of this study, a location for the mobile data collection equipment had to be established within line-of-sight of the surface movement area. Initial feasibility studies were conducted from a car parked in an observation lot at the northeast corner of the airport. Once the data collection method was validated, a more permanent location was secured through the generosity of the Frontiers of Flight Museum, which overlooks the airport from the southeast corner with minimal obstructions. The museum provided a 
secure office with a windowsill for the antenna and power for the various hardware devices. The remote data collection effort lasted over four months and overlapped the purchased PASSUR data.

The remote data collection unit placed at the Frontiers of Flight Museum consisted of multiple hardware devices each serving a specific purpose. The foremost was a Kinetic Avionics SBS-3 ADS-B decoder receiving the transponder messages through a $1090 \mathrm{MHz}$ antenna. The real-time output of this device generally feeds a type of aircraft tracking software, but it also decodes the non-ADS-B Mode S and Mode A/C messages. All decoded messages were streamed to a Raspberry Pi microcomputer where they were timestamped and archived. By definition, this data is intended for real-time use and is not sent with a timestamp. Therefore, each message is associated with a time as it is received by the collecting system. A mobile internet device was also connected to the Raspberry Pi for remote communications with the hardware. The internet connection also kept the recording system in sync with real time, as the internal clocks are prone to drift if not periodically corrected. Original plans were to stream the data live, but data usage limits prevented this from being feasible. Figure 3 shows the hardware used in the mobile data collection unit.

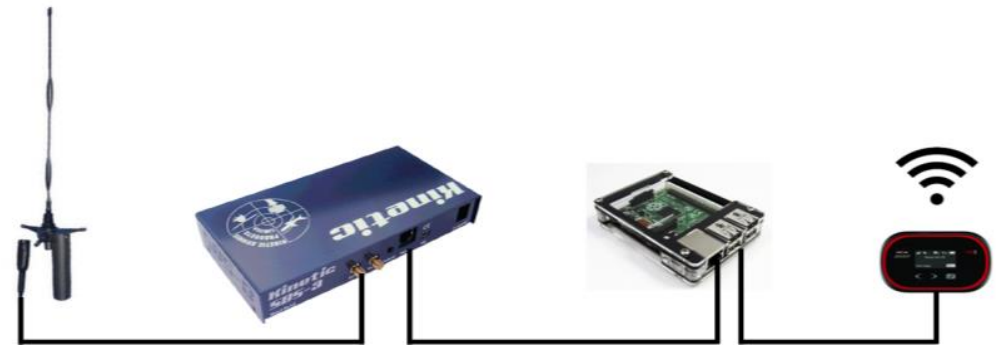

Figure 3. Mobile transponder data collection hardware. From left to right: $1090 \mathrm{MHz}$ antenna, Kinetic SBS-3 transponder decoder, Raspberry Pi computer, and mobile internet device.

\section{Data Analysis Results}

\section{A. Data Validation}

One of the key assumptions in this work was that the PASSUR data was accurate and could be used to validate the Mode A/C and Mode S data that were collected. To confirm this assumption empirically observed departure event times were collected over one day from Dallas Love's tower. The times included pushback, queue entry, runway threshold crossing, and wheels-off times. Of these times, wheels-off contained the least uncertainty in terms of human-error of visually observing the event, so it was used as the heaviest weighted factor when evaluating PASSUR's data. PASSUR's wheels-off times were all within 1 minute of the visually observed times. Other surface movement event times were also well within an acceptably accurate range. As a result, PASSUR data was deemed acceptable to validate other OTA sources and to evaluate any benefit the data could provide beyond that of strictly baseline data.

\section{B. Analysis of Mode A/C and Mode S}

The initial research plan was to use ADS-B to provide surface position data. However, in reality, very few aircraft at DAL broadcast ADS-B. The two remaining OTA broadcasts were Mode A/C and S. Due to the frequency of transmissions, both message types result in a vast quantity of data when logged. Messages are received for all broadcasts within range (arrivals, departures, and en route flights), which means parsing through the data without the knowledge of the call signs of expected departures would be an overwhelming task. Figure 4 tallies the number of Mode A/C messages received for each octal beacon code during a 5-minute period. This figure serves only to illustrate the magnitude of the data being processed and its somewhat unpredictable nature. In order to derive meaning from the data, two key pieces of information are needed: a time (such as a scheduled departure time) and a flight ID (either beacon code or Mode $S$ address).

Knowing the specific beacon code for a flight and the approximate time it is scheduled for pushback greatly reduces the data to manageable levels. Figure 5 shows the number of received Mode A/C transponder messages for a specific beacon code. Data was parsed for beacon code 2234, beginning forty minutes prior to and ten minutes after the scheduled pushback time. There are a few random messages received before minute 46. At first glance, it appears that the number of messages at minute 40 might indicate the pushback of the departure; however, further investigation into other flights has revealed certain levels of vagueness for signals with less than 100 hits per minute. It is only at minute 46 where uncertainty of who is sending the message disappears. Unfortunately, in most cases, this time is within 1 minute of the wheels-off time, which is of limited value when attempting to schedule departures before they depart. 
As noted above, Mode A/C allows for only 4096 unique octal codes (0000 7777), which must be shared between aircraft identification numbers and altitude reporting. This limited range induces uncertainty in the ability to pair received messages with the aircraft that actually sent them. While uncertainty of flight matching exists with Mode A/C identifiers, Mode S addresses are unique for each aircraft. This uniqueness allows a single Mode $\mathrm{S}$ message to be used as a positive identifier for an active aircraft. The four subplots of Figure 6 compare the differences in Mode $\mathrm{A} / \mathrm{C}$ and $\mathrm{S}$ messages with regard to time of positive identification and actual wheels-off time. Both data types tend to have a spike in transmissions at the wheelsoff time, which is of limited value when trying to schedule wheels-off times for departures. The last subplot illustrates a major flaw in using Mode A/C data for novel surveillance. In most cases, 20 or more Mode $\mathrm{A} / \mathrm{C}$ messages per minute indicate a positive identification for a departure; however, in some cases more are needed. The relationship between the minimum threshold of signal transmissions for positive ID and the time difference between wheels-off time and first positive identification is inverse. Twenty messages per minute identify the first aircraft one minute before wheels-off time, but produce a false positive ID for the last aircraft. If the threshold were raised to 100 per minute, the false positives would be eliminated but all ID times would be equal to the departure's wheels-off time.

The benefit of the uniqueness of Mode $S$ addresses is that a single message can be used as a positive identification. As seen in Figure 6, this provides the active state of departures 4 to 11 minutes before wheels-off time. On average the first Mode $\mathrm{S}$ message is received 7 minutes before wheels-off. This is true for both the air carriers and general aviation; however, the variance is greater for general aviation flights. This is expected because all commercial planes operate in a more predictable manner than general aviation, where each flight has its own schedule. Because of the earlier positive identification times, Mode S was chosen over Mode A/C as the superior transponder data for this research.
Number of Transponder Messages
During 5 Minutes on July 16, 2015

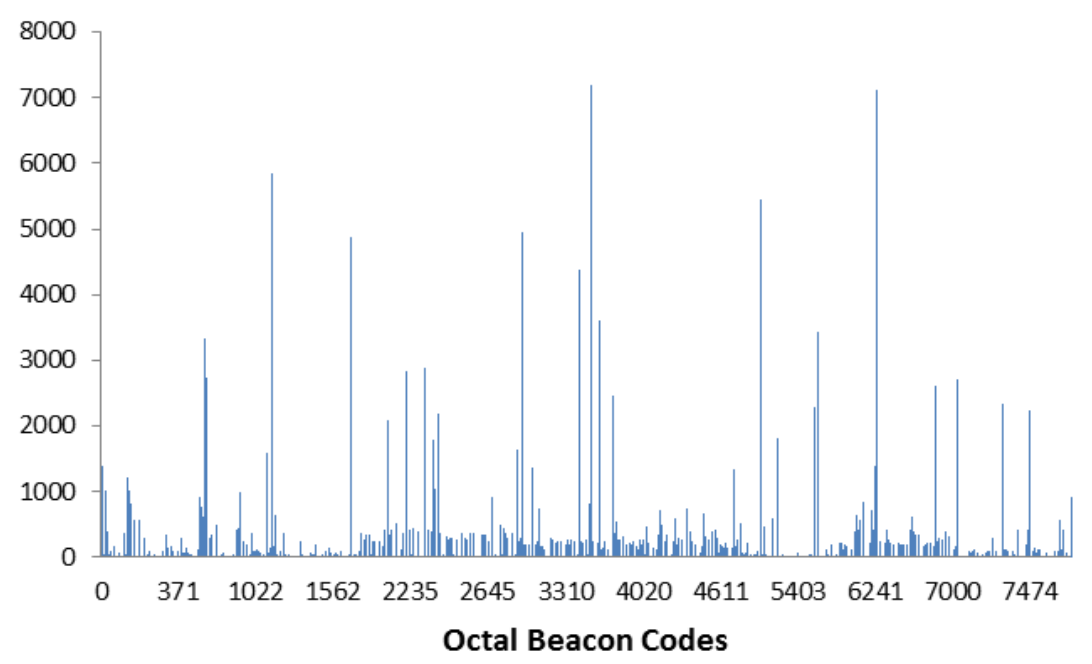

Figure 4. The number of Mode A/C messages received during a 5-minute period for each octal beacon code.

\section{Number of Mode A/C Transmissions for Beacon Code 2234 in Minute Bins}

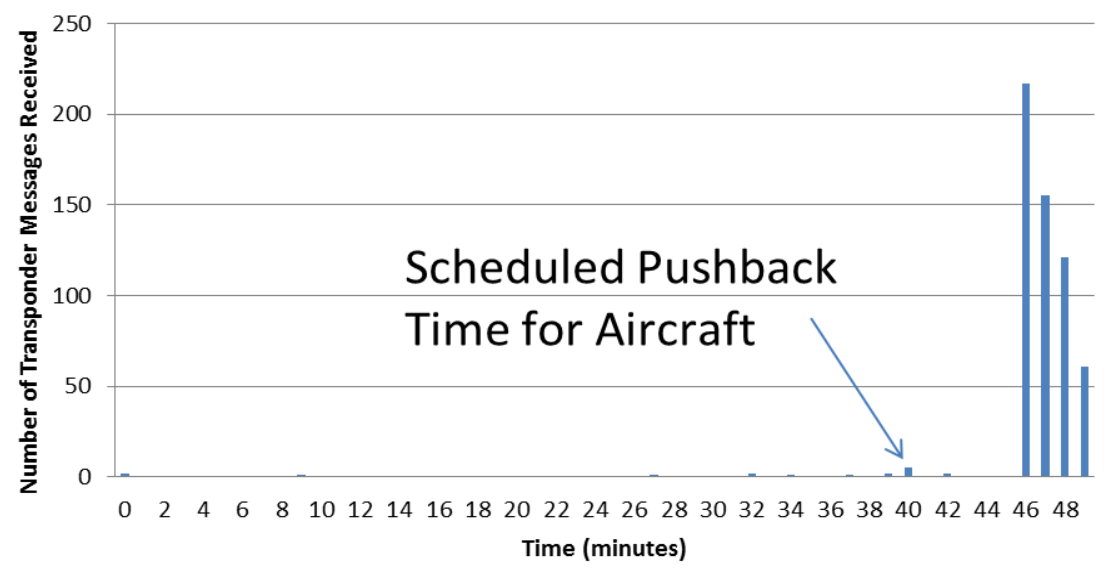

Figure 5. A timeline showing the number of Mode A/C transponder messages for each minute for a single aircraft. 


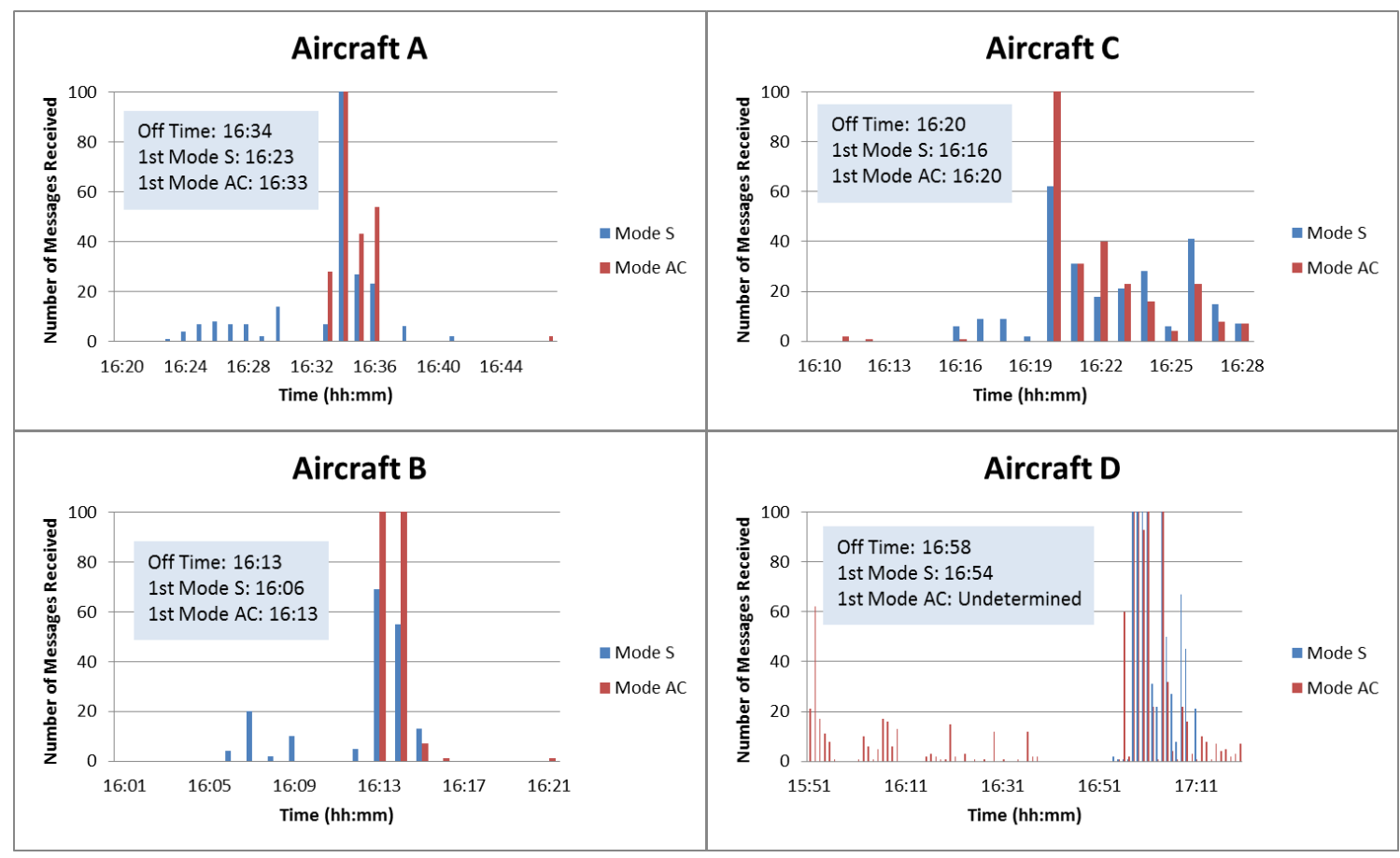

Figure 6. A comparison of Mode $\mathbf{A} / \mathbf{C}$ and Mode $\mathbf{S}$ data. For each subplot, representing a different departure, the number of received messages for each minute of time is tallied. The actual off-time is denoted in addition to the time of first positive identification of the flight for both Mode $S$ and Mode A/C messages. All vertical axes were set to a maximum of 100 messages to aid in readability, however, the number of Mode A/C messages usually spikes above 200.

\section{Comparison of Mode S and PASSUR}

Each data message from PASSUR contains much more information than a Mode $\mathrm{S}$ message. Thus, when comparing the two, we are not referring to the content of the messages but only the time at which the first message was received from a new flight. The time of the first received message is the earliest indication and positive identification that a departure is active on the surface. PASSUR's first message received time and its position data are all based on Mode $\mathrm{S}$ messages. Because of the common base, the OTA first recorded Mode $\mathrm{S}$ message times are very close (less than +/- $1 \mathrm{~min}$ ) to PASSUR's first message times. The slight differences may be attributed to the variations between the placement of PASSUR's multiple antennas and the single antenna of the OTA collection device. The main use of Mode S data for PASSUR at DAL is to find aircraft positons through their multilateration system. Therefore, at least four antennas need to pick up the Mode S message before the first related PASSUR message is generated. PASSUR installed 9 antennas around DAL to ensure full coverage of aircraft surface movement. As discussed in subsequent subsection E, the use of multiple antennas greatly increases the reception of transponder messages and is the main reason why PASSUR usually receives messages slightly before the single Mode $\mathrm{S}$ antenna.

In order to get a better understanding of where the aircraft were when they were first picked up by PASSUR, their positions at that time were plotted on the DAL surface map shown in Figure 7. The color of the markers indicates their relative time before wheels-off, while the shape specifies the air carrier. As can be seen, Southwest Airlines (circular markers) comprises the bulk of the traffic at DAL, with Virgin America (triangular markers) occupying a few gates, and the remainder are mainly general aviation. While the terminal and ramp area serve the commercial planes (center of the airport), the Fixed Base Operators (FBOs), along the perimeter of the airport, serve the general aviation (square markers). In most cases, the commercial planes start broadcasting their Mode $\mathrm{S}$ messages while they are still in the ramp area about 7 minutes before wheels-off time. Aircraft that broadcast their Mode $\mathrm{S}$ messages less than $3 \mathrm{~min}$ before wheels-off time are almost all general aviation flights. This shorter time for some general aviation flights can be attributed to various factors. Their taxi distances are shorter, during the 
prevailing south flow configuration, because of the hangar locations. They also do not generally follow specified procedures like the airlines and there is greater variance associated with when they turn on their transponders.

Figure 7 provides a picture of where the aircraft were when the first Mode S message was received. Without the PASSUR data, the Mode S data collected from one antenna would not provide any location information. Because of the very close correlation of times between PASSUR and the independently collected Mode S data the assumption can be safely made that these positions were representative of the aircraft locations when a first Mode $S$ message was received on a single antenna.

Apart from validating Mode $\mathrm{S}$ data, the PASSUR data has provided insight into the characteristics of departure activity times. Table 2 shows the average times and their standard deviations for two months of PASSUR data. Some outlier data were removed to provide better estimates of standard operations. The outliers were defined as

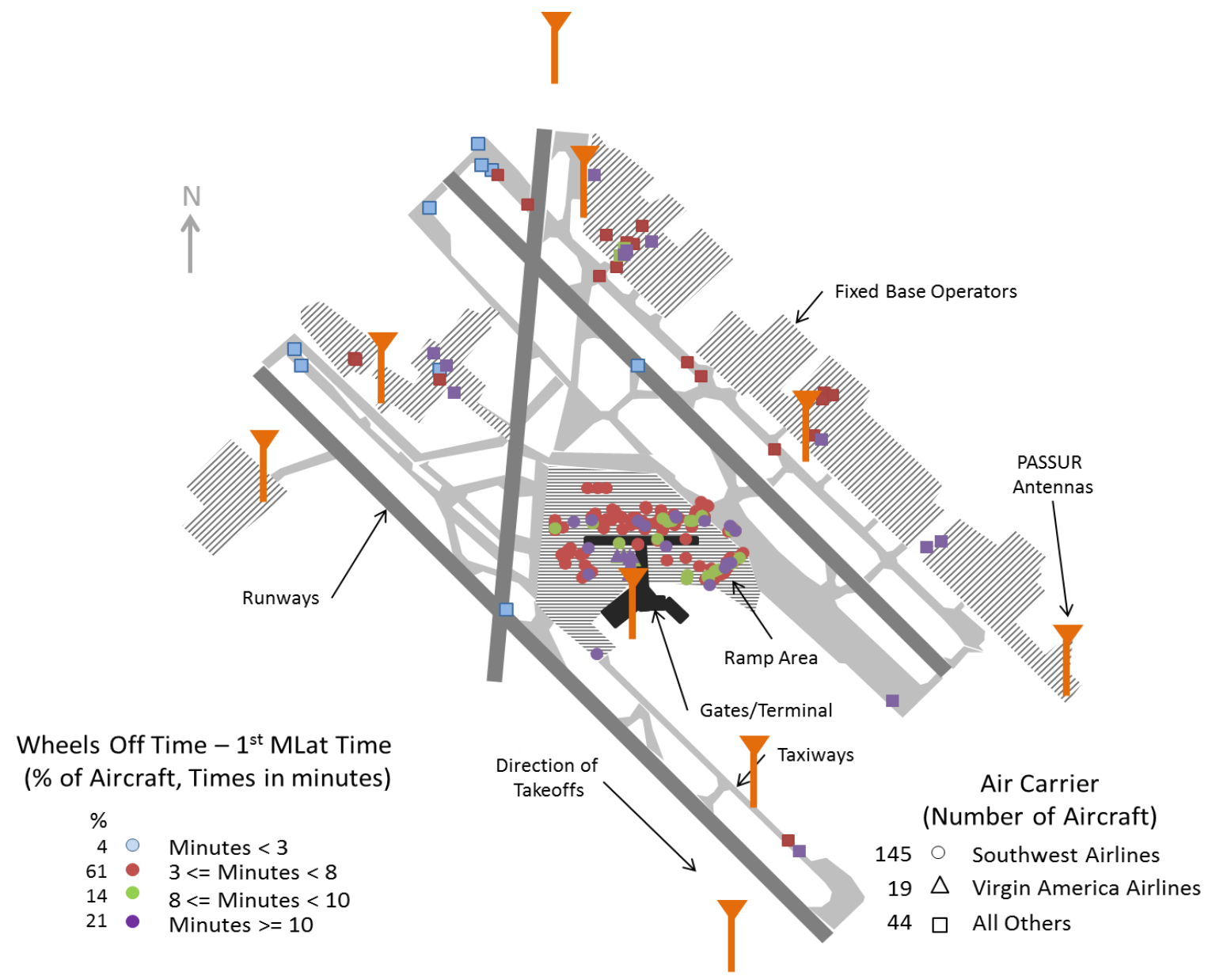

Figure 7. A Dallas Love Field surface map showing where departures were when they were first identified by PASSUR. The airport is in south flow configuration.

Table 2 - PASSUR statistics for two months of data. Actual wheels-off times minus: scheduled pushback, first transponder message, queue entry, and runway entry times. Over 17,000 departures total. All times in minutes.

\begin{tabular}{cc|cc|cc|cc}
\hline \multicolumn{2}{c|}{ ActOff - SchedPush } & \multicolumn{2}{|c|}{ ActOff - 1stTransMsg } & \multicolumn{2}{c|}{ ActOff - QueueEntry } & \multicolumn{2}{c}{ ActOff - RwyEntry } \\
\hline$A v g$ & $S t d D e v$ & $A v g$ & $S t d D e v$ & $A v g$ & StdDev & $A v g$ & StdDev \\
12.34 & 5.89 & 7.24 & 4.04 & 3.43 & 2.04 & 2.09 & 2.10 \\
\hline
\end{tabular}


flights with actual wheels-off time minus the scheduled pushback time greater than 45 minutes. Based on visual observations at DAL, any flight that took longer than 45 minutes to depart encountered some unforeseen abnormal issue. These data can be used to estimate departure event times. While these statistics reflect all the flights at DAL, segregating the flight data by commercial and general aviation will reduce the variance and provide better estimates.

\section{Mode S line-of-light limitations}

The line-of-sight nature of transponder broadcasts has the greatest influence on receiving messages from aircraft on the ground. A receiving antenna must be placed very close to the airport surface; however terminal buildings, hangars, ATC towers, and other obstacles can all eclipse the observed area that an antenna can 'see.' The effect of these obstructions on the reception of transponder messages will impact the usefulness of the data. To evaluate the extent of the delay in message reception at Dallas Love Field, the difference in times between the first Mode $\mathrm{S}$ message and the actual wheels-off time were compared at each gate over four days. The results are shown in Figure 8. The average differences between the first Mode $S$ and actual wheels-off time are plotted radially for each gate. The daily average values for four days are shown by their respective marker shape. The radial lines provide reference from the gate to its average values. For example, gate 13 had three average times of six minutes and one, on July $22^{\text {nd }}$, of about five minutes. In addition to the plotted data, an underlying graphic was added to show the locations of the gates at the terminal and the relative location of the Mode $\mathrm{S}$ antenna.

In theory, departing aircraft within direct line-of-sight of the antenna would be picked up sooner than those eclipsed by an obstruction. The earlier reception of an aircraft's transponder message provides more advanced

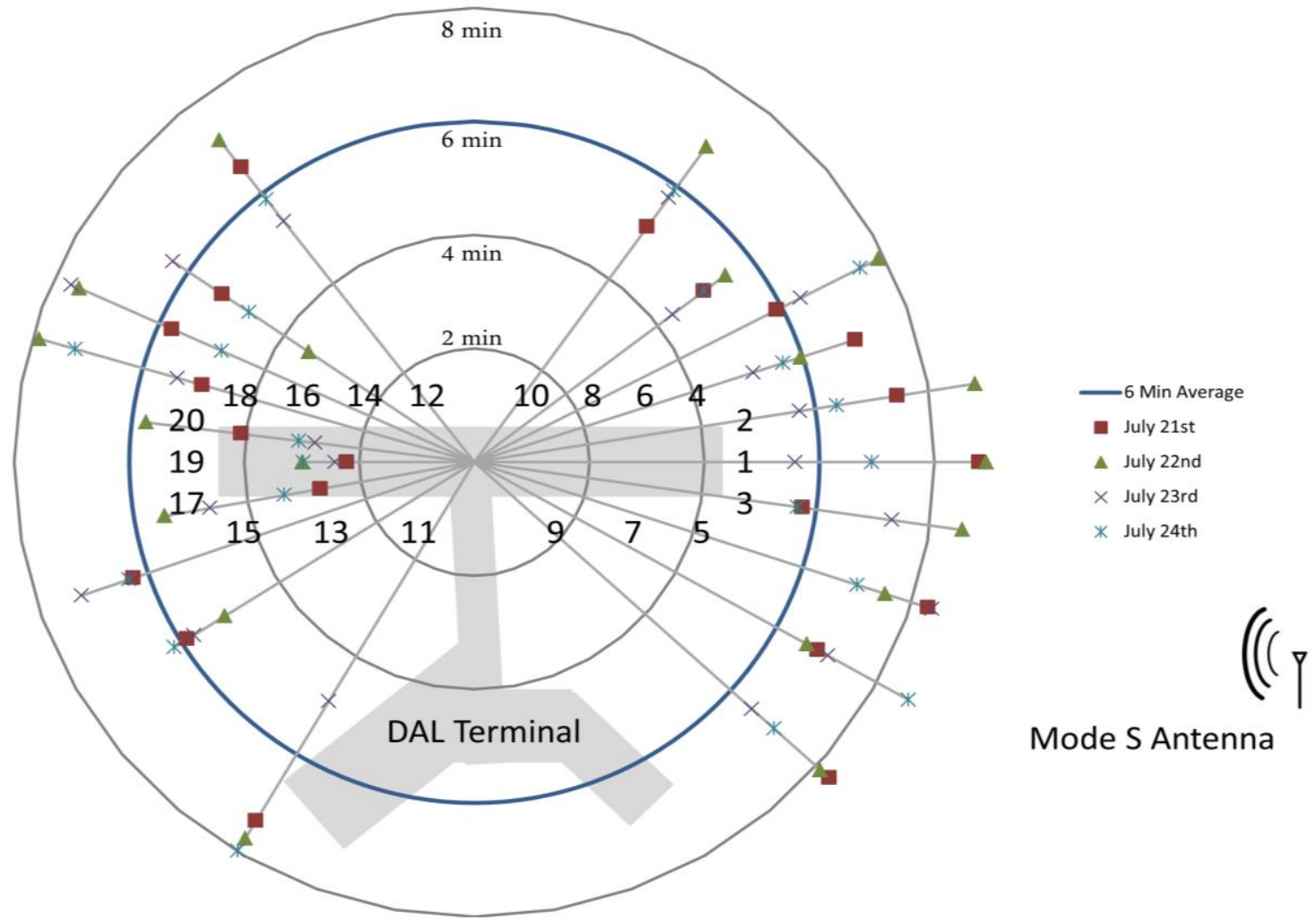

Actual Off Time - First Mode S Message Time (min)

Figure 8. The effects of gate location on the time of first Mode $\mathbf{S}$ message reception relative to the antenna location. For each gate and day, the average difference between the first Mode $S$ message and actual wheels-off time were calculated. The average times were plotted radially over the DAL terminal to show their respective gate location. For purposes of departure scheduling, greater time differences are more desirable as they provide more advance notice time to schedule an aircraft. 
notice time to schedule a departure. The effect of line-of-sight obstruction is most evident in gates 17, 19, and 20 in Figure 8. The first messages from aircraft departing from these gates are received 2-4 minutes later than the messages from aircraft on the opposite side of the terminal. The average times for the partially obscured gates lie between the times of the direct line-of-sight gates and those fully obscured.

These results fit nicely with the multi-antenna PASSUR results from Table 2, where the difference in actual wheels-off time and first PASSUR message time was 7.24 minutes on average for two months of data. The similar average in Figure 8 was less, 6 min, because it focused solely on the departures from the terminal gates which naturally filtered out all of the general aviation. With fewer aircraft in the data set, the lower time differences from gates 17, 19, and 20 had more influence on reducing the average value. Table 3 shows the average and standard deviations for this data set broken out by day. Each day contained between 159 and 179 departures from a terminal gate.

Table 3 - Mode S statistics for the data in Figure 8. The average and standard deviations are for actual wheels-off times minus the first transponder message time for departures from a terminal gate. All times in minutes.

\begin{tabular}{|c|c|c|c|c|c|c|c|}
\hline \multicolumn{2}{|c|}{ July $21^{\text {st }}$} & \multicolumn{2}{|c|}{ July $22^{\text {nd }}$} & \multicolumn{2}{|c|}{ July $23^{\text {rd }}$} & \multicolumn{2}{|c|}{ July $24^{\text {th }}$} \\
\hline$A v g$ & StdDev & $A v g$ & StdDev & $A v g$ & StdDev & $A v g$ & StdDev \\
\hline 5.54 & 3.30 & 6.44 & 4.25 & 5.38 & 3.41 & 5.51 & 3.40 \\
\hline
\end{tabular}

\section{E. ADS-B data evaluation}

Discussions of Mode S data would not be complete without a look at ADS-B messages. Many researchers and plane tracking hobbyists collect ADS-B data and plot tracks of aircraft flying above. The system works best when aircraft are airborne because of the obstruction free line-of-sight, but there are real shortcomings when attempting to use this data for all aircraft and for those on the surface. The first of these shortcomings is compliance. Adoption of ADS-B transponders varies within and amongst air carriers and private planes. DAL averages 285 departures a day. ${ }^{12}$ Of the flights studied, all Virgin America flights broadcast ADS-B data, but they only represent $6 \%$ of the flights at DAL. Southwest Airlines, having the largest presence at DAL at $60 \%$, had only $16 \%$ of its flights equipped with ADS-B. The remaining fleet mix at DAL, consisting mainly of general aviation, had around $20 \%$ compliance. Overall, more than $75 \%$ of the flights operated at DAL do not yet broadcast ADS-B. Until all aircraft filing flight plans are equipped to broadcast ADS-B, the data cannot be used at a system level for surface surveillance and scheduling.

Another limitation of ADS-B data can be found with its usefulness as the sole source of surface data when collected with only one antenna. The entire secondary surveillance system was developed to track airborne aircraft, so attempting to use the data to track surface movement has its issues, such as line-of-sight obstruction and multipath signal propagation errors. The 'A' in ADS-B is for Automatic and refers to the periodic and unsolicited 'squawking' of ADS-B messages from the aircraft. ${ }^{13}$ What is unknown is when the automatic squawking begins and how many of these messages can be successfully collected by a single antenna. As Figure 9 shows, there are gaps in the ADS-B data collected at DAL. The blue track plots are of two aircraft recorded by PASSUR, one departing on runway 13L (northeast of the terminal) and the other on runway 13R (southwest of the terminal). The red plots are the same two aircraft, but the ADS-B track data was collected using the single Mode S antenna. The first set of tracks evaluated were all southwest side departures similar to the one shown below. In every case, there existed a similar gap in the tracks. In some cases, the track data did not start until the aircraft was half way down the runway. The initial suspicion was that the terminal building was casting a shadow over the southwestern runway preventing transponder message reception at the antenna located to the east. This was proven not to be the case once the northeast side tracks were plotted and similar gaps in track data were discovered without an obstruction between the antenna and aircraft. Upon further investigation non-ADS-B Mode S messages were found within the gaps in ADSB track data. This further substantiates the claim that the gaps are not due solely to a line-of-sight obstruction. When comparing the rate of messages received over time, the data in Figure 9 look very similar to the plots in Figure 6 where each flight starts with an initial blip of data followed by a slight lull before a spike in messages and then a steady rate of transmissions. After considering the reception statistics recorded by Hicok and Lee ${ }^{14}$ the gaps in data at DAL appear to be a result of a combination of multipath propagation errors and using a single antenna. Other studies focused on using ADS-B for surface surveillance all used other sources of data in addition to ADS-B and fused the sources together to form a complete picture of the surface movements. ${ }^{14,15,16}$ PASSUR uses a total of nine antennas at DAL, which it uses to collect ADS-B data and also as a basis for its multilateration system. The 
results demonstrated that a single antenna collecting ADS-B is not sufficient to provide usable surface surveillance data.

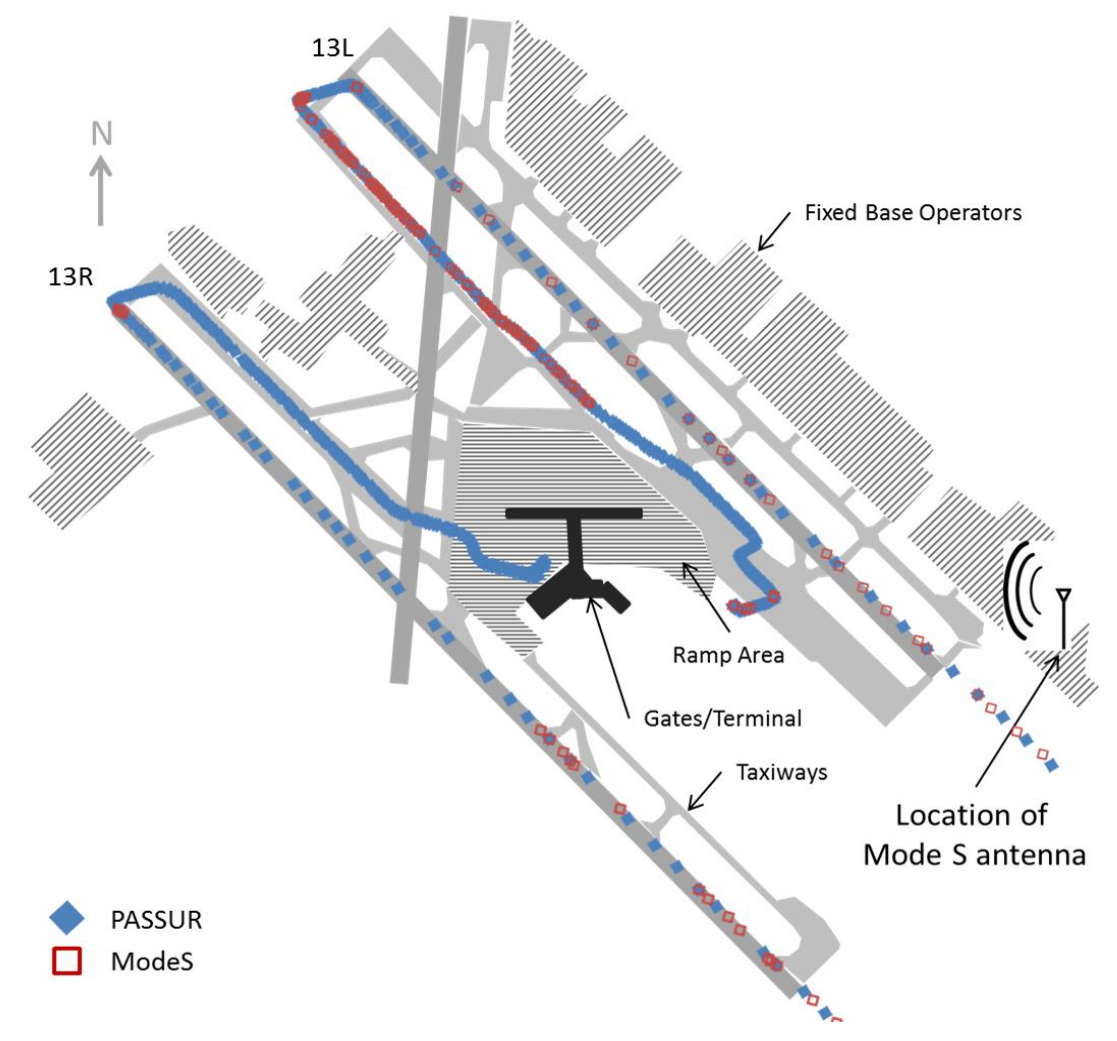

Figure 9. A comparison of track plots between PASSUR and over-the-air collected Mode S ADS-B at DAL. The gaps in the ADS-B track data are clearly seen when compared to the PASSUR tracks.

\section{Conclusions}

The goal of this research was to determine the value of novel surface surveillance at lesser-equipped airports. The value stems from the feasibility of integrating departures from lesser-equipped airports into a tactical departure scheduler along with flights from well-equipped airports using novel surveillance. At Dallas Love Field airport, a single $1090 \mathrm{MHz}$ antenna was demonstrated to be capable of capturing transponder broadcasts for $100 \%$ of the departures based on a complete set of flights provided by the PASSUR data. Messages were received in both Mode $\mathrm{A} / \mathrm{C}$ and Mode $\mathrm{S}$ formats. Mode $\mathrm{A} / \mathrm{C}$ data was less usable than Mode $\mathrm{S}$ because positive aircraft identification was not acquired until just before the aircraft became airborne. Mode $\mathrm{S}$ provided positive identification on average seven minutes before wheels-off. The location of aircraft when their first message was received was generally predictable for commercial air carriers, as they appear to turn their transponders on while still in the ramp area. Time and location of general aviation departures when they engage their transponders are less predictable. This is expected, as general aviation are not bound as tightly to airline schedules and practices.

Compared to Mode S, PASSUR's data was more complete and showed higher fidelity. This is because PASSUR's data is a fusion of multiple data sources and the OTA data is collected using 9 antennas placed around the airport. Surface position data is derived from the transponder broadcasts using multilateration. While position data adds volumes to the surface surveillance picture, the data is only available where the infrastructure has been installed. Within the D10 TRACON, Dallas Love Field is the only airport with PASSUR data. The data were excellent for validating the independently collected Mode S messages at DAL. Because of its limited availability, however, it would not be able to be used to integrate all the lesser-equipped airports into a departure scheduler.

Once all aircraft are equipped and broadcasting ADS-B messages, creating a surface surveillance at lesserequipped airports will be greatly simplified. The current state of ADS-B compliance ( $25 \%$ at DAL), however, is 
insufficient to make this a reality today. Even when all aircraft are ADS-B compliant, a single receiving antenna will not be adequate. The exact number of antennas needed to get reliable coverage of all aircraft is dependent on the size and geometry of the airport. Based on previous studies and PASSUR's infrastructure at DAL, somewhere between five and nine antennas appear to be an acceptable range for high fidelity coverage.

Overall, the Mode S data collected appears to be satisfactory for use as a surrogate surface surveillance, which is used to provide an indication of state of readiness for departures at lesser-equipped airports. Using Mode $\mathrm{S}$ data at DAL, the active state of departures can be determined 4 to 11 minutes before wheels-off time. On average the first Mode S message is received 7 minutes before wheels-off. However, there are some opportunities to improve the accuracy of the Mode S data. The two main areas of uncertainty are length of taxi time and aircraft location when its first transponder message is received. The first is a function of the airport geometry, departure gate, assigned runway, other traffic, etc. and is beyond the scope of this work to change. The second area of uncertainty has potential of being reduced by installing more antennas and aggregating the data feeds into a single source. In addition to evaluating the benefit of using multiple antennas, adding other lesser-equipped airports to the departure scheduler is something that should be pursued in future work.

\section{References}

\footnotetext{
${ }^{1}$ Aponso, B., Coppenbarger, R., Jung, Y., Quon, L., et al, "Identifying Key Issues and Potential Solutions for Integrated Arrival, Departure, Surface Operations by Surveying Stakeholder Preferences”, $15^{\text {th }}$ AIAA Aviation Technology, Integration, and Operations Conference, Dallas, TX, June 22-26, 2015.

${ }^{2}$ Engelland, S., Capps, A, "Trajectory-Based Takeoff Time Predictions Applied to Tactical Departure Scheduling: Concept Description, System Design, and Initial Observations", AIAA 11th Aviation Technology, Integration, and Operations (ATIO) Conference, Virginia Beach, VA, September 20-22, 2011.

${ }^{3}$ Capps, A., Engelland, S, "Characterization of Tactical Departure Scheduling in the National Airspace System," AIAA 11th Aviation Technology, Integration, and Operations (ATIO) Conference, Virginia Beach, Virginia, 20-22 September, 2011.

${ }^{4}$ Engelland, S.A., Capps, A., Day, K., Kistler, M., Gaither, F., and Juro, G., "Precision Departure Release Capability (PDRC) Final Report," NASA/TM-2013-216533, June 2013.

${ }^{5}$ Kistler, M., Capps, A., Engelland, S., "Characterization of Nationwide TRACON Departure Operations," AIAA 14 ${ }^{\text {th }}$ Aviation Technology, Integration, and Operations (ATIO) Conference, Atlanta, GA, 16-20 June 2014.

${ }^{6}$ Capps, A., Kistler, M., Engelland, S., "Design Characteristics of a Terminal Departure Scheduler," AIAA $14^{\text {th }}$ Aviation Technology, Integration, and Operations (ATIO) Conference, Atlanta, GA, 16-20 June 2014.

${ }^{7}$ Budinger, J.,Hall, E., "Aeronautical Mobile Airport Communications System (AeroMACS)," NASA Technical Memorandum 2011-217236, October 2011.

${ }^{8}$ Wanke, C. R., Callaham, M. B., Greenbaum, D. P., and Masalonis, A. J., "Measuring Uncertainty in Airspace Demand Predictions for Traffic Flow Management Applications," Proceedings of the AIAA Guidance, Navigation, and Control Conference, AIAA, Austin, Texas August 2003.

${ }^{9}$ Jeppsen, "RNAV 1 Equipment Notes on U.S. RNAV STAR and SID Procedures and RNAV Operation on RNAV Q and T Routes," Briefing Bulletin JEP 07-A, 9 March 2007.

${ }^{10}$ Federal Aviation Administration, "Automatic Dependent Surveillance-Broadcast (ADS-B) Out Performance Requirements

To Support Air Traffic Control (ATC) Service,” Federal Register, Vol. 75, No. 103, 28 May 2010.

${ }^{11}$ Patel, S., Brinton, C., Gallet, B., Schwartz, B., "The Unmanned Autonomous Aircraft Control System," AIAA Infotech@Aerospace (I@ A) Conference, St. Louis, MO, 29-31 March 2011.

${ }^{12}$ Federal Aviation Administration, "Air Traffic Activity System (ATADS) > Airport Operations," [online database], URL: http://aspm.faa.gov/opsnet/sys/Airport.asp [sited 19 February 2016].

${ }^{13}$ International Standards and Recommended Practices for Aeronautical Telecommunications, Annex 10, Volume IV, ICAO, February 2007.

${ }^{14}$ Hicok, D., Lee, D., “Application of ADS-B for Airport Surface Surveillance,” IEEE, 1998.

${ }^{15}$ Milisavljevic, N., "Sensor and Data Fusion," I-Tech Education and Publishing, Vienna, Austria, 2009, Chap. 6.

${ }^{16}$ Khadilkar, H,. Balakrishnan, H., Reilly, B., "Analysis of Airport Performance using Surface Surveillance Data: A Case Study of BOS," AIAA 11th Aviation Technology, Integration, and Operations (ATIO) Conference, Virginia Beach, Virginia, 2022 September, 2011.
} 